
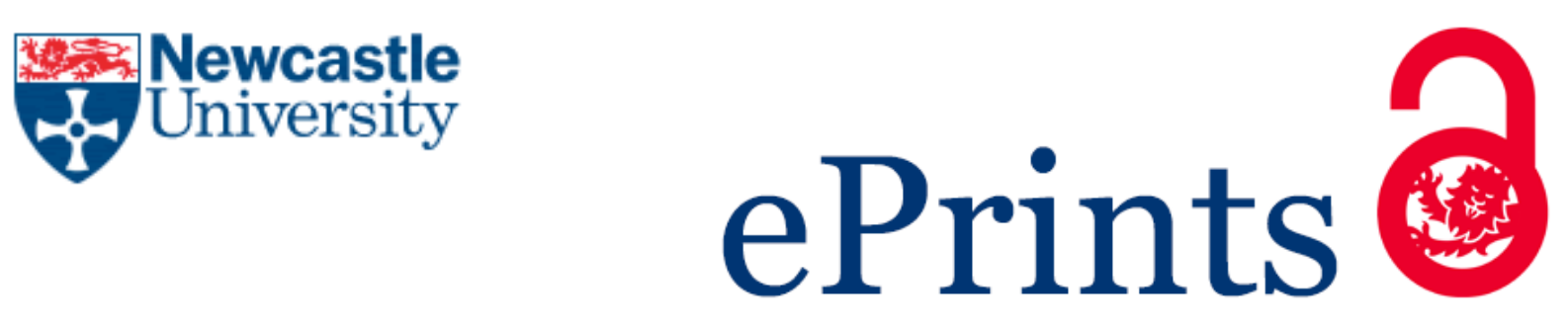

Bayliss HR, Lortie CJ, Stewart GB. How "good" is half a fish? Communicating outcomes of quantitative syntheses to decision makers. Frontiers in Ecology and the Environment 2015, 13(10), 533-534.

\title{
Copyright:
}

Copyright by the Ecological Society of America

DOI link to article:

http://dx.doi.org/10.1890/15.WB.018

Date deposited:

$23 / 03 / 2016$

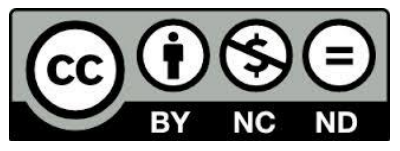

This work is licensed under a

Creative Commons Attribution-NonCommercial-NoDerivatives 4.0 International licence 


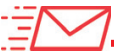

\section{How "good" is half a fish? Communicating outcomes of quantitative syntheses to decision makers}

\section{Peer-reviewed letter}

Syntheses such as meta-analyses and systematic reviews are increasingly being used to inform ecological and environmental science, policy, and practice (Pullin 2012; Koricheva and Gurevich 2014; Lortie 2014). Many incorporate aspects of statistical analysis that allow identification of trends and patterns in the available data (Lortie 2014). In ecological syntheses, the outcomes of individual studies are frequently presented as effect sizes using, for example, standardized mean difference, Pearson correlation coefficient, and response ratios (Gates 2002). Effect sizes allow comparison of different response measures and, when paired with confidence intervals, provide a tool to interpret the strength of outcomes and their variability, including the role of sampling error. Interpreting the meaning of the effect size can be challenging for synthesis authors, let alone for readers. Therefore it is particularly important that it is communicated clearly and accurately; otherwise, decision makers can be left wondering how "good" an increase of half a fish per unit area really is.

Within a synthesis, there are multiple effect sizes that need to be taken into account. To understand how individual studies have contributed to the outcome, decision makers (or their advisors) must consider both the effect sizes of the individual studies - including the number of studies and their relative weight within the analysis - and the overall pooled effect size. In addition, they need to understand the direction of the effect (ie whether it is positive or negative), the magnitude of effect (ie if it is very small, very large, or somewhere in between), and any associated uncertainty. The magnitude of effect can be critical for informing decisions, yet is typically obscured by simplistic vote-counting approaches (Gurevich and Hedges 1999; Nakagawa and Cuthill 2007; Koricheva and Gurevich 2013). Selection of appropriate meta-analysis methodologies is therefore important in ensuring the data generated can usefully inform decisions.

Interpretation of the magnitude of mean effect sizes in ecology appears to conform to coarse benchmarks for other disciplines (with an effect size of 0.2 as small, 0.5 as moderate, and 0.8 as large), and it has been proposed that a similar perspective on assigning relevance should be adopted in future ecological syntheses (Lortie et al. 2015). However, such benchmarks can be misleading, given that statistical significance does not necessarily correlate with biological importance; smaller effect sizes may be ecologically important in complex natural systems (Lortie et al. 2015) whereas statistical significance may simply reflect high statistical power and have limited ecological effect. A pooled effect size can indicate an increase of half a fish per unit area following a management activity and may have little practical application, but a key strength of meta-analysis is the ability to explore reasons for variation between individual studies (at least for any variables for which data exist), facilitating an enhanced understanding of the context in which specific factors can have an effect. Decisions associated with incorporating heterogeneity between studies and what constitutes a substantive effect should be made prior to synthesis and, in many cases, in consultation with relevant stakeholders to avoid biased rationalizations later. For those familiar with the study system, effect sizes in the original units may be more readily interpretable than standardized effect statistics (Nakagawa and Cuthill 2007), but for everyone else it is important that all measures are clearly reported.

Even if a synthesis results in a significant outcome, other factors must be considered when drawing conclusions. For instance, although a syn- thesis on the effectiveness of engineered structures for increasing salmonid abundance generated a statistically significant overall pooled effect size, heterogeneity between included studies and suspected publication bias led the authors to conclude the evidence was equivocal (Stewart et al. 2009). Readers of syntheses must consider the comprehensiveness and appropriateness of the search used to identify relevant studies, any data imputations or manipulations used (both of which require proper reporting to determine), and uncertainties associated with the processes that generated the primary data. The nature of ecological research means that it often takes place in settings where it is difficult to control variables, and multiple methods may be used to test a hypothesis and record or explain natural variation (Koricheva and Gurevich 2014). Because multiple factors act on ecological systems, highly accurate predictions may be infrequent but syntheses will help establish both the common responses and their ranges across systems (Hansson 2003). Conducting sensitivity analyses to link environmental factors with study outcomes will address some of this uncertainty but open data (ie data free to access and reuse) are critical (Hampton et al. 2013). Also, hierarchical statistical methods can account for uncertainties where these can be quantified (Cressie et al. 2009). Frameworks to help establish the value of missing information have been developed in healthcare (eg Claxton 1999) and would also be useful for environmental decision makers to determine which aspects of uncertainty are most critical. Furthermore, simple steps - including carefully defining and reporting the aim of the metaanalysis, identifying which details need to be reported based on the target audience, and considering alternative ways of reporting these results - will facilitate communication to other decision makers and scientists. Consistent, standardized reporting, for example using PRISMA 
reporting standards (Moher et al. 2009), and inclusion of a glossary may help to overcome issues associated with interpreting specialist jargon. Additionally, the use of alternative forms of publication, such as open-access policy briefs, should facilitate access and uptake by nonacademic readers. Importantly, replicable synthesis is thus also possible and this is critical for decision makers and policy. Meanwhile, guidance for grading the quality of evidence and strength of recommendations produced in medicine by the GRADE working group (www.gradeworking group.org) is likely to provide a useful starting point for standardizing communication of limitations and uncertainty in ecological syntheses to decision makers. This may further the development of balanced interpretations and use by the community, particularly if the treatment of primary datasets is well articulated in future syntheses.

Helen R Bayliss ${ }^{1 *}$, Christopher J

Lortie $^{2}$, and Gavin B Stewart ${ }^{3}$

${ }^{1}$ Centre for Evidence-Based

Conservation, School of Environment,

Natural Resources and Geography,

Bangor University, Bangor, UK

*(helenrbayliss@gmail.com);

${ }^{2}$ Department of Biology, York

University, Toronto, Canada;

${ }^{3}$ Centre for Rural Economy, School of

Agriculture, Food and Rural

Development, Newcastle University,

Newcastle upon Tyne, UK

Claxton K. 1999. The irrelevance of inference: a decision-making approach to the stochastic evaluation of health care technologies. J Health Econ 18: 341-64.

Cressie N, Calder CA, Clark JS, et al. 2009. Accounting for uncertainty in ecological analysis: the strengths and limitations of hierarchical statistical modeling. Ecol Appl 19: 553-70.

Gates S. 2002. Review of methodology of quantitative reviews using meta-analysis in ecology. J Animal Ecol 71: 547-57.

Gurevich J and Hedges LV. 1999. Statistical issues in ecological metaanalysis. Ecology 80: 1142-49.

Hampton SE, Strasser CA, Tewksbury JJ, et al. 2013. Big data and the future of ecology. Front Ecol Environ 11: 156-62.

Hansson L. 2003. Why ecology fails at application: should we consider vari- ability more than regularity? Oikos 100: 624-27.

Koricheva J and Gurevich J. 2013. Place of meta-analysis among other methods of research synthesis. In: Koricheva J, Gurevich J, and Mengersen K (Eds). Handbook of meta-analysis in ecology and evolution. Princeton, NJ: Princeton University Press.

Koricheva J and Gurevich J. 2014. Uses and misuses of meta-analysis in plant ecology. J Ecol 102: 828-44.

Lortie CJ. 2014. Formalized synthesis opportunities for ecology: systematic reviews and meta-analyses. Oikos 123: 897-902.

Lortie CJ, Stewart GB, Rothstein H, and Lau J. 2015. How to critically read ecological meta-analyses. Res Syn Methods 6: 124-33.

Moher D, Liberati A, Tetzlaff J, et al. 2009. Preferred reporting items for systematic reviews and meta-analyses: the PRISMA statement. PLoS Med 6: e1000097.

Nakagawa S and Cuthill IC. 2007. Effect size, confidence interval and statistical significance: a practical guide for biologists. Biol Rev 82: 591-605.

Pullin AS. 2012. Realising the potential of environmental data: a call for systematic review and evidence synthesis in environmental management. Environ Evid 1:2.

Stewart GB, Bayliss HR, Showler DA, et al. 2009. Effectiveness of engineered instream structure mitigation measures to increase salmonid abundance: a systematic review. Ecol Appl 19: 931-41.

doi:10.1890/15.WB.018

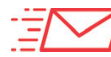

\section{Not quite free yet: clarifying UAV regulatory progress for ecologists}

Following our previous letter (Front Ecol Environ 13[2]: 74-75) on regulatory hurdles associated with adding Unmanned Aerial Vehicles (UAVs) to ecological research, Allan et al. (Front Ecol Environ 13[7]: 354-55) provided a timely response that demonstrates the current confusion surrounding new and proposed US Federal Aviation Administration (FAA) regulation of UAV use for non-recreational purposes. While UAV laws in the US are changing, the cost for an ecologist to become a "certified operator" as Allan et al. suggest remains high, given that at a minimum, one must become a licensed sport pilot (FAA 2015a). This training costs thousands of dollars and covers aeronautical informa- tion (AOPA 2015) well beyond what is necessary to safely operate UAVs.

Section 333 of The FAA Modernization Act of 2012 (FAA 2012) sets forth guidelines for UAV use during their integration into the National Airspace System (ie all facilities, airspace, craft, and pilots involved in civil and military aviation), and generally prohibits UAV use except for purely recreational purposes. The FAA is currently granting Section 333 exemptions on a case-by-case basis; receiving this exemption gives operators blanket authorization to fly small UAVs for non-recreational purposes at or below 200 feet anywhere in the US. However, it is still necessary to hold a current Certificate of Authorization (COA) in order to operate a UAV above 200 feet or in the vicinity of airports or using published instrument flight procedures (ie established routes used by manned aircraft in conditions of reduced visibility) (FAA 2015a, b). All flights made under Section 333 exemptions must still have a certified pilot at the controls and the UAV must be registered with the FAA. Section 333 exemptions have been granted to some US universities, as Allan et al. mention, but the majority of exemptions have been made to private companies charging for their services, thus creating a new pay-for-UAV market that is inaccessible to ecologists with low operating budgets.

Allan et al. suggest partnering with a "registered industry partner" as a convenient solution to regulatory hurdles. While this is a valid suggestion, it requires ecologists using UAVs to pay external companies to perform flights and provide UAV equipment. This is akin to renting a tool with considerable restrictions, not the incorporation of a new approach into a researcher's toolbox. Everyone on our team is capable of safely programming and flying UAV missions and troubleshooting UAV hardware issues; all are well versed in FAA regulations. These skills were gained through careful self-training, as are most new skills acquired by 